\title{
Atributos biológicos indicadores da qualidade do solo em pastagem cultivada e nativa no Pantanal
}

\author{
Evaldo Luis Cardoso(1), Marx Leandro Naves Silva(2), Fátima Maria de Souza Moreira(2) e Nilton Curi(2)
}

(1)Embrapa Pantanal, Caixa Postal 109, CEP 79320-900 Corumbá, MS. E-mail: evaldo@cpap.embrapa.br (2)Universidade Federal de Lavras, Departamento de Ciência do Solo, Caixa Postal 3.037, CEP 37200-000 Lavras, MG. E-mail: marx@ufla.br, fmoreira@ufla.br, niltcuri@ufla.br

\begin{abstract}
Resumo - O objetivo deste trabalho foi avaliar as alterações nos atributos biológicos do solo, em razão da conversão da floresta nativa em pastagem cultivada e da submissão de pastagens nativas a sistema de pastejo contínuo, em um Neossolo Quartzarênico, no Pantanal sul-mato-grossense. O trabalho consistiu da avaliação de três florestas nativas; três pastagens de Brachiaria decumbens com diferentes idades de formação, implantadas em substituição às florestas nativas; e três pastagens nativas (uma sob sistema de pastejo contínuo e duas sem pastejo por 3 e 19 anos). Foram coletadas amostras de solo nas profundidades $0-10$ e $10-20 \mathrm{~cm}$, em três transectos de $100 \mathrm{~m}$ estabelecidos em cada ambiente de estudo, cada um dos quais constituiu uma repetição. Os atributos avaliados foram: carbono orgânico total (COT), carbono microbiano (Cmic), quociente microbiano $(q \mathrm{MIC})$, respiração basal $(\mathrm{RB})$ e quociente metabólico $\left(q \mathrm{CO}_{2}\right)$. A substituição da floresta nativa por pastagem cultivada promoveu redução nos teores de COT, Cmic e $q \mathrm{MIC}$ e elevação da RB; o sistema de pastejo contínuo em pastagem nativa promoveu redução nos teores de COT e no Cmic. O Cmic é o atributo mais sensível às alterações impostas ao solo, pelos sistemas de pastagens cultivada e nativa, pois foi marcado por reduções mais expressivas.
\end{abstract}

Termos para indexação: atividade microbiana, carbono microbiano, quociente metabólico, quociente microbiano, respiração basal.

\section{Biological indicator attributes of soil quality under cultivated and natural pasture in the Pantanal wetlands}

\begin{abstract}
The objective of this work was to evaluate the changes in the biological attributes of soil promoted by substitution of the native forest by cultivated pasture, and by the submission of native pasture under continuous grazing in the Pantanal wetlands. The work consisted of the evaluation of three native forests, three Brachiaria decumbens pastures of different formation ages, established in substitution to the native forests and three native pastures, one under continuous grazing and two without grazing for 3 and 19 years. Soil samples were collected at $0-10$ and $10-20 \mathrm{~cm}$ depths, in the three 100-m transects in each sampled site. Each transect represented one replicate. The following attributes were evaluated: total organic carbon (TOC), soil microbial carbon (Cmic), microbial quotient $(q \mathrm{MIC})$, basal respiration (BR) and metabolic quotient $\left(q \mathrm{CO}_{2}\right)$. The substitution of the native forest by cultivated pasture reduced TOC, $\mathrm{Cmic}$, and $q \mathrm{MIC}$ and increased $\mathrm{BR}$. The continuous grazing on native pasture reduced TOC and Cmic contents. Cmic was the most sensitive attribute to the alterations imposed on soil by the cultivated and native pasture systems, since it was marked by more substantial reductions.

Index terms: microbial activity, microbial biomass carbon, metabolic quotient, microbial quotient, basal respiration.
\end{abstract}

\section{Introdução}

Com a globalização da economia e com os mercados competitivos, o Pantanal - que tem na pecuária de corte a sua principal atividade econômica - tem enfrentado grande pressão política, econômica e social pela necessidade de compatibilizar aumentos de produtividade com a conservação ambiental. A busca por aumentos de produtividade da pecuária pantaneira tem levado a desmatamentos para a implantação de pastagem cultivada, o que tem despertado preocupação quanto à sustentabilidade dos agroecossistemas do Pantanal, pois essas ações, de maneira geral, são conduzidas sem se considerarem as características peculiares dos distintos ambientes que compõem a paisagem $\mathrm{e}$, invariavelmente, tendem a contribuir para o desequilíbrio ambiental, e nem sempre resultam em aumentos de produtividade. 
Ambientes de floresta nativa, notadamente aqueles estabelecidos em solos de baixa fertilidade natural, como é o caso do Pantanal, têm sua manutenção fortemente associada ao equilíbrio entre a cobertura vegetal e os processos biogeoquímicos do solo. De acordo com Moreira \& Malavolta (2004), a produtividade dos ecossistemas naturais e de agroecossistemas introduzidos e raramente fertilizados depende da reciclagem dos nutrientes minerais, contidos na serapilheira das plantas e da matéria orgânica do solo.

O grau de impacto da remoção da floresta nativa está relacionado ao manejo do sistema produtivo e das práticas agrícolas (Silva et al., 2007). Nas últimas décadas, a avaliação da qualidade do solo tem merecido destacada atenção, e a quantificação de alterações nos seus atributos, decorrentes da intensificação de sistemas de uso e manejo, tem sido amplamente realizada para monitorar a produção sustentável dos solos (Neves et al., 2007) e, consequentemente, a conservação dos recursos naturais. Como a microbiota do solo é a principal responsável pela decomposição dos compostos orgânicos, pela ciclagem de nutrientes e pelo fluxo de energia do solo, a biomassa microbiana e sua atividade têm sido apontadas como as características mais sensíveis às alterações na qualidade do solo, causadas por mudanças de uso e práticas de manejo (Trannin et al., 2007).

Segundo Gama-Rodrigues \& Gama-Rodrigues (2008), a biomassa microbiana pode ser enquadrada como o compartimento central do ciclo do $\mathrm{C}$ e representa considerável reservatório de nutrientes nos solos e atributo fundamental para o estudo de ciclagem de nutrientes, em diferentes ecossistemas. De acordo com Chaer \& Tótola (2007), os indicadores microbiológicos podem ser de grande importância, na avaliação precoce de eventuais efeitos adversos do manejo sobre a qualidade do solo, o que permite a adoção antecipada de medidas corretivas ou de controle, além de permitir identificar o que ocorre com o sistema de manejo em curso, ou seja, se contribui para aumentar ou diminuir a sustentabilidade do sistema de produção. Embora as alterações nos atributos biológicos sejam relevantes na avaliação da qualidade do solo, a maioria dos estudos tem sido sobre o efeito de sistemas de manejo em culturas anuais ou florestais nos latossolos do Cerrado, porém são escassos os resultados em outros importantes biomas brasileiros que elucidam alterações decorrentes da conversão de floresta nativa em pastagem cultivada.
O objetivo deste trabalho foi avaliar as alterações na biomassa e atividade microbiana do solo, em razão da conversão da floresta nativa em pastagem cultivada e da submissão de pastagens nativas a sistema de pastejo contínuo, em um Neossolo Quartzarênico, no Pantanal sul-mato-grossense.

\section{Material e Métodos}

O estudo foi realizado em duas fazendas, na sub-região da Nhecolândia, cujo clima é tropical subúmido (Aw, Köppen), com temperatura média anual de $26^{\circ} \mathrm{C}$ e precipitação média de 800 a $1.200 \mathrm{~mm}$ (Cadavid Garcia, 1986). Os ambientes de estudo consistiram de três diferentes florestas nativas; três pastagens cultivadas, implantadas em substituição às florestas nativas; e três pastagens nativas, caracterizadas pela predominância de Mesosetum chaseae e Axonopus purpusii, em diferentes sistemas de pastejo. Os ambientes avaliados foram: FN (floresta nativa), mata semidecídua $\left(18^{\circ} 34^{\prime} 57^{\prime \prime} \mathrm{S}\right.$ e $\left.55^{\circ} 50^{\prime} 52^{\prime \prime} \mathrm{W}\right)$, com ocorrência de 24 diferentes espécies, com altura média de $12,4 \mathrm{~m}$ e diâmetro médio de $27 \mathrm{~cm}$, em que Attalea phalerata, Rhamnidium elaeocarpum e Astronium fraxinifolium se destacam como mais abundantes (Salis, 2004); PC27, pastagem cultivada de Brachiaria decumbens, com 27 anos de formação, implantada em substituição e adjacente à FN; CE1, cerradão $\left(18^{\circ} 33^{\prime} 11^{\prime \prime S}\right.$ e $\left.55^{\circ} 48^{\prime} 41^{\prime \prime} \mathrm{W}\right)$, com ocorrência de 30 diferentes espécies, com altura média de $11,7 \mathrm{~m} \mathrm{e}$ diâmetro médio de $15,8 \mathrm{~cm}$, em que Qualea grandiflora, Caryocar brasiliense e Lafoensia pacari se destacam como mais abundantes (Salis, 2004); PC26, pastagem cultivada de Brachiaria decumbens, com 26 anos de formação, implantada em substituição e adjacente ao CE1; CE2, cerradão (1859'57"S e 56 $\left.38^{\circ} 10^{\prime \prime} \mathrm{W}\right)$, com ocorrência de 43 diferentes espécies, com altura média de 7,5 m e diâmetro médio de $12,4 \mathrm{~cm}$, em que se destacam como mais abundantes Alibertia sessilis, Protium heptaphyllum e Zanthoxylum rigidum (Salis, 2004); PC11, pastagem cultivada de Brachiaria decumbens, com 11 anos de formação, implantada em substituição e adjacente ao CE2; PNpc, pastagem nativa submetida ao sistema de pastejo contínuo $\left(18^{\circ} 59^{\prime} 25^{\prime \prime} \mathrm{S}\right.$ e $\left.56^{\circ} 38^{\prime} 43^{\prime \prime} \mathrm{W}\right)$; PNv3, pastagem nativa sem pastejo por três anos $\left(18^{\circ} 59^{\prime} 57^{\prime \prime} \mathrm{S}\right.$ e $\left.56^{\circ} 38^{\prime} 1^{\prime \prime} \mathrm{W}\right)$; PNv19, pastagem nativa sem pastejo por 19 anos $\left(18^{\circ} 58^{\prime} 42^{\prime \prime} \mathrm{S}\right.$ e $56^{\circ} 37^{\prime} 0^{\prime \prime} \mathrm{W}$ ), área instituída como Reserva Particular do Patrimônio Natural (RPPN). 
As pastagens cultivadas foram implantadas após a derrubada e queima da floresta nativa e tanto esta quanto aquelas jamais receberam qualquer tipo de correção ou adubação do solo. A lotação animal nessas pastagens, conforme a intensidade e duração da cheia, variam de 0,8 a $1 \mathrm{UA} \mathrm{ha}^{-1}$, na pastagem cultivada (Crispim et al., 2004), e de 0,2 a $0,5 \mathrm{UA} \mathrm{ha}^{-1}$, na pastagem nativa (Santos et al., 2003). O solo dos ambientes de estudo é o Neossolo Quartzarênico órtico, enquadrado na classe textural areia.

A amostragem do solo, realizada no final do período de seca, consistiu da realização de três transectos de $100 \mathrm{~m}$, em cada ambiente de estudo, e de coleta de amostras compostas de cinco subamostras (a cada $20 \mathrm{~m}$ ) à profundidade de $0-10$ e de $10-20 \mathrm{~cm}$. Cada transecto constituiu uma repetição no total de 27 transectos. As amostras foram acondicionadas em sacos de plástico, protegidas da luz e mantidas em caixas térmicas até a chegada ao laboratório; em seguida, foram peneiradas ( $2 \mathrm{~mm}$ de malha), com a umidade ajustada para 50 a $60 \%$ da capacidade de campo, acondicionadas em sacos de plástico com furos para ventilação e mantidas em câmara fria $\left(4^{\circ} \mathrm{C}\right)$.

$\mathrm{O}$ carbono da biomassa microbiana (Cmic) foi avaliado pelo método da fumigação-extração (Vance et al., 1987), que consiste na extração do Cmic, após a aplicação de clorofórmio às amostras, o que provoca morte dos microrganismos e liberação dos componentes celulares. O quociente microbiano ( $q \mathrm{MIC}$ ), ou relação Cmic/COT, foi calculado de acordo com Sparling (1992). A respiração basal (RB) foi determinada pelo $\mathrm{CO}_{2}$ evoluído a partir de $20 \mathrm{~g}$ de solo, incubado durante 72 horas, extraído com solução de $\mathrm{NaOH} 0,05 \mathrm{~mol} \mathrm{~L}^{-1}$ e titulado com $\mathrm{HCl} 0,05 \mathrm{~mol} \mathrm{~L}^{-1}$ (Isermeyer, 1952). $\mathrm{O}$ quociente metabólico $\left(q \mathrm{CO}_{2}\right)$ foi calculado pela razão entre a respiração basal e o Cmic (Anderson \& Domsch, 1993), expresso em microgramas de $\mathrm{C}-\mathrm{CO}_{2}$ por micrograma de Cmic por dia. O carbono orgânico total (COT) foi determinado conforme Yeomans \& Bremner (1988), cujo princípio é a oxidação a quente com dicromato de potássio e titulação do dicromato remanescente com sulfato ferroso amoniacal. Todas as amostras foram analisadas em triplicatas.

O delineamento experimental foi o inteiramente casualizado, com estrutura em faixas, em razão das profundidades de amostragem. A análise de variância foi realizada com os procedimentos do Sisvar (Ferreira, 2000). As comparações para verificação do efeito da conversão da floresta nativa em pastagem cultivada e do sistema de pastejo contínuo em pastagem nativa, em relação à pastagem sem pastejo por 3 e 19 anos, foram realizadas por meio de contrastes ortogonais, a partir do desdobramento dos oito graus de liberdade dos ambientes de estudo. A significância dos contrastes de interesse, com um grau de liberdade, foi testada pelo teste $\mathrm{F}$, a $5 \%$ de probabilidade, tendo-se considerado o quadrado médio do resíduo obtido pela análise de variância.

\section{Resultados e Discussão}

Os teores de carbono orgânico total (COT) e carbono microbiano ( $\mathrm{Cmic}$ ) foram significativamente alterados pela conversão da floresta nativa em pastagem cultivada e pelo sistema de pastejo contínuo da pastagem nativa, notadamente na camada superficial, onde é mais intensa a atividade da microbiota do solo (Tabela 1). A substituição da floresta nativa por pastagem cultivada promoveu reduções nos teores de COT e Cmic, respectivamente, de 25 e $42 \%$ na PC27, 41 e $53 \%$ na PC26 e 21 e $42 \%$ na PC11. Na pastagem nativa submetida ao sistema de pastejo contínuo, em comparação à pastagem sem pastejo por 19 anos, ocorreu redução, na camada de $0-10 \mathrm{~cm}$, de $36 \%$ nos teores de COT e $51 \%$ no Cmic; não foi constatada

Tabela 1. Carbono orgânico total (COT), carbono microbiano (Cmic), e quociente microbiano ( $q \mathrm{MIC}$ ), em amostras do solo de diferentes ambientes no Pantanal sul-mato-grossense, e valores de $\mathrm{F}$ dos contrastes de interesse.

\begin{tabular}{|c|c|c|c|c|c|c|}
\hline \multirow{2}{*}{$\begin{array}{l}\text { Ambiente }^{(1)} \mathrm{e} \\
\text { contraste }\end{array}$} & \multicolumn{2}{|c|}{$\operatorname{COT}\left(\mathrm{g} \mathrm{kg}^{-1}\right)$} & \multicolumn{2}{|c|}{$\operatorname{Cmic}\left(\mu \mathrm{g} \mathrm{g}^{-1}\right)$} & \multicolumn{2}{|c|}{$q \mathrm{MIC}(\%)$} \\
\hline & $0-10 \mathrm{~cm}$ & $10-20 \mathrm{~cm}$ & $0-10 \mathrm{~cm} 1$ & $0-20 \mathrm{~cm}$ & $0-10 \mathrm{~cm}$ & $10-20 \mathrm{cr}$ \\
\hline$\overline{\mathrm{FN}}$ & 15,8 & 11,2 & 486 & 320 & 3,2 & 2,9 \\
\hline CE1 & 11,3 & 3,4 & 339 & 89 & 3,1 & 2,7 \\
\hline CE2 & 7,6 & 4,0 & 184 & 84 & 2,5 & 2,2 \\
\hline $\mathrm{PC} 27$ & 11,9 & 7,8 & 280 & 154 & 2,4 & 2,1 \\
\hline $\mathrm{PC} 26$ & 6,6 & 2,7 & 159 & 56 & 2,4 & 2,1 \\
\hline $\mathrm{PC} 11$ & 6,0 & 3,4 & 106 & 92 & 1,7 & 2,1 \\
\hline $\mathrm{PNpc}$ & 4,6 & 3,0 & 90 & 68 & 1,9 & 2,3 \\
\hline PNv3 & 4,4 & 2,6 & 104 & 84 & 2,3 & 2,8 \\
\hline \multirow[t]{2}{*}{ PNv19 } & 7,2 & 5,2 & 184 & 98 & 2,2 & 2,4 \\
\hline & \multicolumn{6}{|c|}{ Valor de F } \\
\hline $\mathrm{FN} \times$ & $28,53 * *$ & $22,61 *$ & $47,36 * *$ & $29,99 * *$ & $4,81^{*}$ & $2,36^{\mathrm{ns}}$ \\
\hline CE1 x PC26 & $42,78^{* *}$ & $0,91^{\mathrm{ns}}$ & $36,28 * *$ & $1,23^{\mathrm{ns}}$ & $3,44 *$ & $1,28^{\mathrm{ns}}$ \\
\hline CE2 x PC11 & $4,89^{*}$ & $0,57^{\mathrm{ns}}$ & $6,68^{*}$ & $0,07^{\mathrm{ns}}$ & $2,96^{*}$ & $0,02^{\mathrm{ns}}$ \\
\hline PNv3 x PNpc & $0,06^{\mathrm{ns}}$ & $0,05^{\mathrm{ns}}$ & $0,22^{\text {ns }}$ & $0,26^{\mathrm{ns}}$ & $0,57^{\mathrm{ns}}$ & $2,35^{\mathrm{ns}}$ \\
\hline PNv19 x PNp & $13,96^{* *}$ & $9,90 * *$ & $4,30 *$ & $0,94^{\mathrm{ns}}$ & $0,04^{\mathrm{ns}}$ & $0,79^{\text {ns }}$ \\
\hline \multicolumn{7}{|c|}{$\begin{array}{l}\text { (1)FN, mata semidecídua; CE1, cerradão; CE2, cerradão; PC27, PC26, PC11, } \\
\text { pastagem cultivada com } 27,26 \text { e } 11 \text { anos de formação, respectivamente; } \\
\text { PNpc, PNv3, PNv19, pastagem nativa com sistema de pastejo contínuo e } \\
\text { sem pastejo por } 3 \text { e } 19 \text { anos, respectivamente. * e **Significativo a } 5 \text { e } 1 \% \\
\text { de probabilidade, respectivamente. }{ }^{*} \text { Não significativo. }\end{array}$} \\
\hline
\end{tabular}

Pesq. agropec. bras., Brasília, v.44, n.6, p.631-637, jun. 2009 
diferença significativa em relação à pastagem sem pastejo por três anos.

Os maiores teores de COT e Cmic observados em FN, CE1, CE2 e PNv19, quando comparados com os respectivos ambientes submetidos ao pastejo, refletiram uma condição mais favorável à microbiota do solo, nos ambientes caracterizados pelo mínimo de intervenção antrópica e em pressuposto equilíbrio. Contrariamente, Carneiro et al. (2008) relataram aumento de aproximadamente $50 \%$ no valor de Cmic, em ambientes de pastagem de Brachiaria decumbens e integração lavoura-pecuária em relação ao cerrado nativo, o que foi atribuído ao sistema radicular fasciculado da gramínea, que se concentra nos primeiros $10 \mathrm{~cm}$ de profundidade e resulta em maior entrada de carbono no solo, via rizosfera e necromassa que atuam na ativação da microbiota do solo. Contudo, de acordo com Bayer \& Mielniczuk (2008), o conteúdo de matéria orgânica do solo, sob vegetação natural, encontra-se estável, e o uso agrícola altera esse conteúdo, em que se observa, normalmente redução acentuada, quando utilizados métodos de preparo com intenso revolvimento do solo e sistemas com baixa adição de resíduos vegetais. No presente trabalho, os ambientes não sofreram revolvimento do solo, por isso, os resultados estão relacionados ao maior aporte contínuo e variado de substrato orgânico, com diferentes graus de suscetibilidade à decomposição, provenientes de vegetação com maior diversidade de espécies (D'Andréa et al., 2002; Gama-Rodrigues et al., 2008), além da grande quantidade de raízes e maior retenção de água no solo (Perez et al., 2004).

Os teores de COT e Cmic na PC27, superiores aos observados nas demais pastagens, e até mesmo semelhantes aos das florestas CE1 e CE2, notadamente na camada de $0-10 \mathrm{~cm}$, indicam que as condições remanescentes da mata semidecídua (FN), ambiente original onde foi implantada a PC27, podem ainda estar prevalecendo na área de pastagem, mesmo após 27 anos de formação. Cerri et al. (1996), ao avaliar a dinâmica de carbono em solos da Amazônia, observaram que, mesmo após oito anos do desmatamento e implantação da pastagem, ainda havia $50 \%$ do carbono do solo proveniente da mata original, o que demonstra que grande parte da matéria orgânica da floresta, a depender do tipo de manejo adotado, permanece sob forma estável. Costa (2005), ao estudar solo de tabuleiro no sul da Bahia, relatou também que, mesmo após 28 anos de implantação de pastagem, foram observados valores médios de 55,76 e $79 \%$ de carbono derivado da mata original, nas camadas de 5, 15 e $30 \mathrm{~cm}$, respectivamente.

Em relação às pastagens nativas, o período de três anos sem pastejo foi insuficiente para elevação dos teores de COT e para melhoria das condições da microbiota do solo, pois não foram constatadas diferenças significativas entre as pastagens $\mathrm{PNpc}$ e PNv3. Esses resultados, aliados aos fatos de a textura ser arenosa e de haver baixa fertilidade natural dos solos, refletem a baixa capacidade de os campos naturais em pastejo contínuo formarem biomassa aérea e depositarem substratos orgânicos na superfície do solo. Em geral, os campos naturais são submetidos à permanente desfolha, imposta pela pressão de pastejo, o que caracteriza esses ambientes como sistemas marcados apenas pela extração de biomassa e, basicamente, sem nenhuma reposição. Entretanto, é importante ressaltar que esses resultados foram constatados em pastagem nativa livre de inundação, o que pode ser diferente na pastagem nativa sujeita à inundação periódica, principalmente de origem fluvial, que também ocorre em expressivas áreas no Pantanal.

No presente trabalho, os valores de Cmic, observados nos ambientes de pastagens cultivada e nativa, foram expressivamente inferiores aos relatados na literatura para pastagens em Latossolo Vermelho-Amarelo, Latossolo Vermelho e Neossolo Quartzarênico (Souza et al., 2006; Araújo et al., 2007; Carneiro et al., 2008; Gama-Rodrigues etal., 2008). Contudo, como a biomassa microbiana responde intensamente às flutuações sazonais de umidade e temperatura, ao cultivo e ao manejo de resíduos (Gama-Rodrigues \& Gama-Rodrigues, 2008), os menores valores podem estar associados tanto à época de amostragem, realizada no final do período de seca, como à provável maior degradação do solo nesses ambientes de pastagem no Pantanal.

Em geral, tanto na conversão da floresta nativa em pastagem cultivada, quanto no sistema de pastejo contínuo da pastagem nativa, as reduções nos valores de Cmic foram mais acentuadas que as dos teores de COT. Portanto, o Cmic, que representa a parte viva e mais ativa da matéria orgânica do solo, é mais sensível à remoção da cobertura vegetal nativa do que a parte não viva da matéria orgânica, razão pela qual é apontado como um indicador de qualidade do solo (Matsuoka et al., 2003). De acordo com 
Tótola \& Chaer (2002), as avaliações do Cmic e sua atividade podem revelar mudanças no conteúdo de matéria orgânica, antes que elas possam ser detectadas na matéria orgânica total, o que possibilita a adoção de medidas corretivas, antes que a perda de qualidade do solo seja mais severa.

De acordo com o quociente microbiano ( $q \mathrm{MIC}$ ), relação que expressa quanto do carbono orgânico do solo está imobilizado na biomassa microbiana, a menor eficiência dos microrganismos na imobilização do carbono ocorreu nas pastagens cultivadas, principalmente na camada superficial, sem diferença significativa nos sistemas de pastejo da pastagem nativa (Tabela 1). A conversão da floresta nativa em pastagem cultivada promoveu reduções no $q \mathrm{MIC}$, à profundidade $0-10 \mathrm{~cm}$, de aproximadamente $25 \%$ na PC27, 22\% na PC26 e 32\% na PC11. De acordo com Gama-Rodrigues \& Gama-Rodrigues (2008), em solos com matéria orgânica de baixa qualidade nutricional, a biomassa microbiana encontra-se sob estresse e é incapaz de utilizar totalmente o $\mathrm{C}$ orgânico e, nesse caso, o $q \mathrm{MIC}$ tende a diminuir. Assim, os menores valores observados no solo sob pastagens cultivadas (PC27, PC26, PC11) indicam uma provável condição de estresse para a população microbiana, decorrente da menor cobertura vegetal e, principalmente da redução da quantidade e qualidade de substrato orgânico na formação de serapilheira. Segundo Gama-Rodrigues \& Gama-Rodrigues (2008), em solos com baixos teores de matéria orgânica, como o caso dos Neossolos no Pantanal, maior ênfase deveria ser dada ao estudo da biomassa microbiana da serapilheira e de seus índices, para melhor avaliação da disponibilidade de carbono no ecossistema.

Em relação à respiração basal $(\mathrm{RB})$ e ao quociente metabólico $\left(q \mathrm{CO}_{2}\right)$, componentes da taxa de respiração específica da biomassa e atributos relacionados ao ciclo do carbono orgânico, embora tenha ocorrido tendência de maior liberação de $\mathrm{C}-\mathrm{CO}_{2}$ nos ambientes sob maior intervenção antrópica, ou seja, nas pastagens cultivadas, implantadas em substituição às floresta nativas (Tabela 2), aumentos significativos foram constatados somente na PC27 $(10-20 \mathrm{~cm})$ e PC11 $(0-10 \mathrm{~cm})$. Resultados de maior atividade microbiana em ambientes de pastagem cultivada, em relação à vegetação nativa, têm sido relatados na literatura (Xavier et al., 2006; Araújo et al., 2007; Carneiro et al., 2008) e atribuídos ao intenso desenvolvimento e ciclagem do sistema radicular das gramíneas forrageiras, na camada superior do solo. Segundo Islam \& Weil (2000), altas taxas de respiração podem indicar tanto um distúrbio ecológico como um alto nível de produtividade do ecossistema. De acordo com Tótola \& Chaer (2002), um baixo $q \mathrm{CO}_{2}$ indica economia na utilização de energia e, supostamente, reflete um ambiente mais estável ou mais próximo do seu estado de equilíbrio; ao contrário, valores elevados são indicativos de ecossistemas submetidos a alguma condição de estresse ou de distúrbio. Contudo, no presente trabalho, diferentemente do COT, Cmic e $q \mathrm{MIC}, \mathrm{o} q \mathrm{CO}_{2}$ não foi um indicador sensível para refletir uma condição adversa para a biomassa microbiana, nos ambientes de pastagem. Não foram constatadas diferenças significativas nos valores de $q \mathrm{CO}_{2}$, nos ambientes sob ação antrópica (PC27, PC26, $\mathrm{PC} 11, \mathrm{PNpc})$, quando comparados com os respectivos ambientes de referência (FN, CE1, CE2, PNv19), exceção feita ao maior valor na $\mathrm{PC} 11$ em relação à CE2 $(0-10 \mathrm{~cm})$, significativo a $5 \%$ de probabilidade. Souza et al. (2008), em sistema de integração agriculturapecuária submetido a diferentes intensidades de pastejo, também não encontraram efeito significativo sobre o $q \mathrm{CO}_{2}$, atribuído à baixa percentagem de biomassa microbiana do solo catabolicamente ativa.

Tabela 2. Respiração basal e quociente metabólico, em amostras do solo de diferentes ambientes no Pantanal sulmato-grossense, e valores de F dos contrastes de interesse.

\begin{tabular}{|c|c|c|c|c|}
\hline \multirow[t]{2}{*}{$\begin{array}{l}\text { Ambiente }^{(1)} \mathrm{e} \\
\text { contraste }\end{array}$} & \multicolumn{2}{|c|}{$\begin{array}{c}\text { Respiração basal } \\
\left(\mu \mathrm{g} \mathrm{C}-\mathrm{CO}_{2} \mathrm{~g}^{-1} \mathrm{dia}^{-1}\right)\end{array}$} & \multicolumn{2}{|c|}{$\begin{array}{c}\text { Quociente metabólico } \\
\left(\mu \mathrm{g} \mathrm{C}-\mathrm{CO}_{2} \mu \mathrm{g}^{-1} \mathrm{Cmic} \mathrm{dia}^{-1}\right)\end{array}$} \\
\hline & $0-10 \mathrm{~cm}$ & $10-20 \mathrm{~cm}$ & $0-10 \mathrm{~cm}$ & $10-20 \mathrm{~cm}$ \\
\hline$\overline{\mathrm{FN}}$ & 22 & 21 & 0,04 & 0,07 \\
\hline CE1 & 23 & 24 & 0,06 & 0,27 \\
\hline CE2 & 18 & 28 & 0,09 & 0,35 \\
\hline $\mathrm{PC} 27$ & 24 & 34 & 0,08 & 0,23 \\
\hline PC26 & 28 & 26 & 0,18 & 0,49 \\
\hline PC11 & 35 & 28 & 0,35 & 0,30 \\
\hline $\mathrm{PNpc}$ & 16 & 16 & 0,18 & 0,30 \\
\hline PNv3 & 17 & 16 & 0,16 & 0,18 \\
\hline \multirow[t]{2}{*}{ PNv19 } & 16 & 25 & 0,11 & 0,33 \\
\hline & \multicolumn{4}{|c|}{ Valor de F } \\
\hline $\mathrm{FN} \times \mathrm{PC} 27$ & $0,31^{\mathrm{ns}}$ & $9,45^{* *}$ & $0,07^{\mathrm{ns}}$ & $1,21^{\mathrm{ns}}$ \\
\hline CE1 x PC26 & $4,75^{\mathrm{ns}}$ & $0,35^{\mathrm{ns}}$ & $0,60^{\mathrm{ns}}$ & $2,17^{\mathrm{ns}}$ \\
\hline CE2 x PC11 & $17,62 * *$ & $0,00^{\mathrm{ns}}$ & $2,93^{*}$ & $0,10^{\text {ns }}$ \\
\hline PNv3 x PNpc & $0,03^{\mathrm{ns}}$ & $0,05^{\mathrm{ns}}$ & $0,01^{\mathrm{ns}}$ & $0,60^{\mathrm{ns}}$ \\
\hline PNv19 x PNpc & $0,00^{\mathrm{ns}}$ & $4,20 *$ & $0,24^{\mathrm{ns}}$ & $0,03^{\mathrm{ns}}$ \\
\hline
\end{tabular}

${ }^{(1)} \mathrm{FN}$, mata semidecídua; CE1, cerradão; CE2, cerradão; PC27, PC26, PC11, pastagem cultivada com 27, 26 e 11 anos de formação, respectivamente; PNpc, PNv3, PNv19, pastagem nativa com sistema de pastejo contínuo e sem pastejo por 3 e 19 anos, respectivamente. * e **Significativo a 5 e $1 \%$

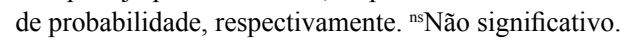




\section{Conclusões}

1. A substituição da floresta nativa por pastagem cultivada reduz os teores de carbono orgânico total, carbono microbiano e o quociente microbiano e eleva a respiração basal.

2. O sistema de pastejo contínuo da pastagem nativa reduz os teores de carbono orgânico total e carbono microbiano, e três anos sem pastejo são insuficientes para a melhoria desses atributos.

3. O carbono microbiano é o atributo mais sensível às alterações no solo, promovidas pela substituição da floresta nativa por pastagem cultivada e pelo sistema de pastejo contínuo em pastagem nativa, no Pantanal.

\section{Referências}

ALEF, K.; NANNIPIERI, P. (Ed.). Methods in applied soil microbiology and biochemistry. London: Academic Press, 1995. $576 \mathrm{p}$.

ANDERSON, T.H.; DOMSCH, K.H. The metabolic quotient for $\mathrm{CO}_{2}\left(q \mathrm{CO}_{2}\right)$ as a specific activity parameter to assess the effects of environmental conditions, such as $\mathrm{pH}$, on the microbial biomass of forest soils. Soil Biology \& Biochemistry, v.25, p.393-395, 1993.

ARAÚJO, R.; GOEDERT, W.J.; LACERDA, M.P.C. Qualidade de um solo sob diferentes usos e sob cerrado nativo. Revista Brasileira de Ciência do Solo, v.31, p.1099-1108, 2007.

BAYER, C.; MIELNICZUK, J. Dinâmica e função da matéria orgânica. In: SANTOS, G. de A.; SILVA, L.S. da; CANELLAS, L.P.; CAMARGO, F.A. de O. (Ed.). Fundamentos da matéria orgânica do solo em ecossistemas tropicais e subtropicais. 2.ed. rev. e atual. Porto Alegre: Metrópole, 2008. p.7-18.

CADAVID GARCIA, E.A. Estudo técnico-econômico da pecuária bovina de corte do Pantanal Mato-Grossense. Corumbá: Embrapa-CPAP, 1986. 150p. (Embrapa-CPAP. Documentos, 4).

CARNEIRO, M.A.C.; ASSIS, P.C.R.; MELO, L.B. de C.; PEREIRA, H.S.; PAULINO, H.B.; SILVEIRA NETO, A.N. da. Atributos bioquímicos em dois solos de cerrado sob diferentes sistemas de manejo e uso. Pesquisa Agropecuária Tropical, v.38, p.276-283, 2008.

CERRI, C.C.; BERNOUX, M.; VOLKOFF, B.; MORAES, J.L. Dinâmica do carbono nos solos da Amazônia. In: ALVAREZ VENEGAS, V.H.; FONTES, L.E.F.; FONTES, M.P.F. (Ed.). Os solos nos grandes domínios morfoclimáticos do Brasil e o desenvolvimento sustentado. Viçosa: Sociedade Brasileira de Ciência do Solo: UFV, 1996. p.61-69.

CHAER, G.M.; TÓTOLA, M.R. Impacto do manejo de resíduos orgânicos durante a reforma de plantios de eucalipto sobre indicadores de qualidade do solo. Revista Brasileira de Ciência do Solo, v.31, p.1381-1396, 2007.

COSTA, O.V. Estoque de carbono e indicadores da qualidade de solo de tabuleiro sob pastagem no sul da Bahia. 2005. 64p. Tese (Doutorado) - Universidade Federal de Viçosa, Viçosa.
CRISPIM, S.M.A.; POTT, A.; RODRIGUES, C.A.G.; CARDOSO, E.L.; COMSTRI FILHO, J.A.; SANTOS, S.A. Pastagem cultivada. In: CARDOSO, E.L. (Ed.). Gado de corte no Pantanal: o produtor pergunta, a Embrapa responde. Brasília: Embrapa Informação Tecnológica, 2004. p.120-128.

D'ANDRÉA, A.F.; SILVA, M.L.N.; CURI, N.; SIQUEIRA, J.O.; CARNEIRO, M.A.C. Atributos biológicos indicadores da qualidade do solo em sistemas de manejo na Região do Cerrado no sul do Estado de Goiás. Revista Brasileira de Ciência do Solo, v.26, p.913-923, 2002.

FERREIRA, D.F. Análises estatísticas por meio do SISVAR (sistema para análise de variância) para Windows versão 4.0. In: REUNIÃO ANUAL DA REGIÃO BRASILEIRA DA SOCIEDADE INTERNACIONAL DE BIOMETRIA, 45., 2000, São Carlos. Anais. São Carlos: Universidade Federal de São Carlos, 2000. p.255-258.

GAMA-RODRIGUES, E.F. da; GAMA-RODRIGUES, A.C. da. Biomassa microbiana e ciclagem de nutrientes. In: SANTOS, G. de A.; SILVA, L.S. da; CANELLAS, L.P.; CAMARGO, F.A. de O. (Ed.). Fundamentos da matéria orgânica do solo: ecossistemas tropicais e subtropicais. 2.ed. rev. e atual. Porto Alegre: Metrópole, 2008. p.159-170.

GAMA-RODRIGUES, E.F. da; GAMA-RODRIGUES, A.C. da; PAULINO, G.M.; FRANCO, A.A. Atributos químicos e microbianos de solos sob diferentes coberturas vegetais no norte do Estado do Rio de Janeiro. Revista Brasileira de Ciência do Solo, v.32, p.1521-1530, 2008.

ISERMEYER, H. Eine einfache Methode zur Bestimmung der Bodenatmung und der Karbonate im Boden. Z. Pflanzenernäh Bodenk, v.56, p.26-38, 1952.

ISLAM, K.R.; WEIL, R.R. Land use effects on soil quality in a tropical forest ecosystem of Bangladesh. Agriculture Ecosystems and Environment, v.79, p.9-16, 2000.

MATSUOKA, M.; MENDES, I.C.; LOUREIRO, M.F. Biomassa microbiana e atividade enzimática em solos sob vegetação nativa e sistemas agrícolas anuais e perenes na região de Primavera do Leste (MT). Revista Brasileira de Ciência do Solo, v.27, p.425-433, 2003.

MOREIRA, A.; MALAVOLTA, E. Dinâmica da matéria orgânica e da biomassa microbiana em solo submetido a diferentes sistemas de manejo na Amazônia Ocidental. Pesquisa Agropecuária Brasileira, v.39, p.1103-1110, 2004.

NEVES, C.M.N. das; SILVA, M.L.N.; CURI, N.; CARDOSO, E.L.; MACEDO, R.L.G.; FERREIRA, M.M.; SOUZA, F.S. de. Atributos indicadores da qualidade do solo em sistema agrossilvopastoril no noroeste do Estado de Minas Gerais. Scientia Forestalis, n.74, p.45-53, 2007.

PEREZ, K.S.S.; RAMOS, M.L.G.; McMANUS, C. Carbono da biomassa microbiana em solo cultivado com soja sob diferentes sistemas de manejo nos Cerrados. Pesquisa Agropecuária Brasileira, v.39, p.567-573, 2004.

SALIS, S.M. Distribuição das espécies arbóreas e estimativa da biomassa aérea de savanas florestadas, Pantanal da 
Nhecolândia, Estado do Mato Grosso do Sul. 2004. 63p.Tese (Doutorado) - Universidade Estadual Paulista, Rio Claro.

SANTOS, S.A.; ABREU, U.G.P. de; CRISPIM, S.M.A.; PADOVANI, C.R.; SORIANO, B.M.A.; CARDOSO, E.L.; MORAES, A.S. Simulações de estimativa de capacidade de suporte das áreas de campo limpo da sub-região da Nhecolândia, Pantanal. Corumbá: Embrapa Pantanal, 2003. 22p. (Embrapa Pantanal. Boletim de Pesquisa e Desenvolvimento, 52).

SILVA, M.B. da; KLIEMANN, H.J.; SILVEIRA, P.M.; LANNA, A.C. Atributos biológicos do solo sob influência da cobertura vegetal e do sistema de manejo. Pesquisa Agropecuária Brasileira, v.42, p.1755-1761, 2007.

SOUZA, E.D. de; CARNEIRO, M.A.C.; PAULINO, H.B.; SILVA, C.A.; BUZETTI, S. Frações do carbono orgânico, biomassa e atividade microbiana em um Latossolo Vermelho sob cerrado submetido a diferentes sistemas de manejo e usos do solo. Acta Scientiarum. Agronomy, v.28, p.323-329, 2006.

SOUZA, E.D. de; COSTA, S.E.V.G. de A.; LIMA, C.V.S. de; ANGHINOMI, I.; MEURER, E.J.; CARVALHO, P.C. de F. Carbono orgânico e fósforo microbiano em sistema de integração agricultura-pecuária submetido a diferentes intensidades de pastejo em plantio direto. Revista Brasileira de Ciência do Solo, v.32, p.1273-1282, 2008.
SPARLING, G.P. Ratio of microbial biomass carbon to soil organic carbon as a sensitive indicator of changes in soil organic matter. Australian Journal of Soil Research, v.30, p.195-207, 1992.

TÓTOLA, M.R.; CHAER, G.M. Microrganismos e processos microbiológicos como indicadores da qualidade do solo. In: ALVAREZ VENEGAS, V.H.; SCHAEFER, C.E.G.R.; BARROS, N.F. de; MELLO, J.W.V. de; COSTA, L.M. da. (Ed.). Tópicos em ciência do solo. Viçosa: Sociedade Brasileira de Ciência do Solo, 2002. p.195-276.

TRANNIN, I.C. de B.; SIQUEIRA, J.O.; MOREIRA, F.M. de S. Características biológicas do solo indicadoras de qualidade após dois anos de aplicação de biossólido industrial e cultivo de milho. Revista Brasileira de Ciência do Solo, v.31, p.1173-1184, 2007.

VANCE, E.D.; BROOKES, P.C.; JENKINSON, D.S. An extraction method for measuring soil microbial biomass C. Soil Biology and Biochemistry, v.19, p.703-707, 1987.

XAVIER, F.A.S.; MAIA, S.M.F.; OLIVEIRA, T.S.; MENDONÇA, E.S. Biomassa microbiana e matéria orgânica leve em solos sob sistemas agrícolas orgânico e convencional na Chapada da Ibiapaba. Revista Brasileira de Ciência do Solo, v.30, p.247-258, 2006.

YEOMANS, J.C.; BREMNER, J.M. A rapid and precise method for routine determination of organic carbon in soil. Communications in Soil Science and Plant Analysis, v.19, p.1467-1476, 1988.

Recebido em 8 de dezembro de 2008 e aprovado em 25 de maio de 2009 